\title{
A method of visualization of a brain neural pathway by using critical points and target regions
}

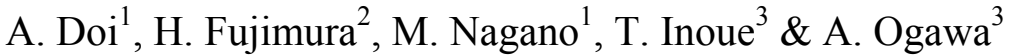 \\ ${ }^{1}$ Iwate Prefectural University, Japan \\ ${ }^{2}$ Alps Corporation, Japan \\ ${ }^{3}$ Iwate Medical University, Japan
}

\begin{abstract}
Visualization of a brain neural pathway is considered as a useful tool for the support of surgery planning. In this paper, we propose a method of visualization of the brain neural pathway by using both critical points and target regions, in order to extract a necessary pathway. Here, a critical point is a vortex center, and we suppose that a neural pathway is a vortical flow field, and we find starting points of streamlines by classifying critical points in the flow field. The user specifies the target regions, such as ROI (Region Of Interest), and visualizes important streamlines that pass through the target regions.

Keywords: visualization, brain neural pathway, DT-MRI, critical points, target regions
\end{abstract}

\section{Introduction}

Brain neural pathway visualization using diffusion tensor-magnetic resonance imaging (DT-MRI) is used in the early detection of illnesses, and in the support of both surgery and post-surgical planning. DT-MRI produces multiple 3dimensional images by applying magnetic fields from multiple directions (6 directions in this research). Pathways such as brain white matter control the movement of water molecules, and signal strength changes according to relationships between magnetic field gradient in the diffusion direction and direction of the pathway. Water molecule diffusion perpendicular to the direction of the white matter pathway is low in comparison to diffusion in a parallel direction $[1,2]$. 
A frequent method of visualizing neural pathways using diffusion direction is to assume that the diffusion is a flow field, displayed through streamlines or arrow diagrams [3-7]. Several major differences from flow fields of fluid dynamics have been identified:

1) Discontinuities exist in flow fields when neural pathways intersect.

2) Anatomically, the important neural pathways within the brain must be shown.

3) Greater distortions and errors exist near bones, which are less affected by magnetic fields, and

4) Many discontinuous voxels appear due to noise in DT-MRI.

In response, we improve the previous methods of calculating critical points for target vortex center [9-11], which have used in computational fluid dynamics fields in the past, in order to apply visualization of brain neural pathways.

More concretely:

- Start points of streamlines are calculated by considering FA (Fractional Anisotropy) values of point groups near critical points, in order to identify appropriate start points.

- From streamlines that pass near critical points, those passing through important regions are extracted and colored appropriately to clearly display the directions of neural pathways.

- In addition to plane or rectangular regions, volume regions can be specified such as the cerebral peduncle, which is important in anatomy.

Normally, specialized knowledge is required to interactively select the start points of streamlines, such as when displaying neural pathways of grey and white matter. This interactive operation is also extremely time-consuming. In addition, when following pathways from the specified start point region, a number of similar pathways will be extracted, making important pathways difficult to distinguish. Furthermore, when neural pathways separate in multiple directions from the starting point voxel, pathways can generally only be followed in a single direction.

To resolve these issues, end points (critical points) of the neural pathway are automatically calculated first. Next, streamlines passing near those end points are calculated, and only those streamlines passing through the target region are displayed (extracted).

Setting target regions for streamlines to pass through is performed ahead of time by the user, by extracting target areas through a segmentation method. Next, each neural pathway that passes through each target region is colored. In this paper, Section 2 discusses the extraction of neural pathways and critical points, Section 3 deals with algorithms to follow neural pathways, Section 4 presents applications and evaluation of this method, and Section 5 covers the conclusions and future issues.

\section{Critical points and neural pathways}

Generally, the direction of neural pathways is stored as tensor data after being deduced by diffusion strength for each direction from MRI and DT-MRI signals. 
This data is diagonalized, and from the obtained 3 eigenvalues, we can presume that the eigenvector with the greatest eigenvalue represents the main direction of the neural pathway [8]. At this point, there is no indication if the eigenvector is in a positive or negative direction, so both possibilities must be considered.

To display streamlines, the user first selects a start point, then follows the streamline from that point. To follow the streamline, tri-linear interpolation of the 8 vertices of the voxel to which the targeted point belongs is calculated [9]. End points are determined when a predetermined number of steps are reached, or the direction value becomes a threshold value (such as $1^{-7}$ ), or error processing is continued for a predetermined number.

Starting points are selected interactively, and the necessary pathways are extracted. To display pursuit results, fractional anisotropy (FA) values, a measure of the degree of diffusion, are used to color-code pathways. FA values range between 0.0 and 1.0, representing degree of anisotropy, with higher values representing higher diffusion differences in each direction.

In the visualization of flow fields, locations of vortex centers (where speed $=$ 0 ) offer an important guideline. Eigenvalues of diffusion tensor in each voxel are obtained from DT-MRI, and the eigenvector for the highest eigenvalue of this group is calculated. This eigenvector can be assumed to be the direction of flow, allowing critical points to be identified. In the critical points calculation used in this paper, the voxel displaying vector values with 8 vertices is divided into 5 tetrahedrons, and location of the vortex center for each tetrahedron is calculated. This calculation does not require the Newton-Raphson Method or other numeric solutions, instead using a cubic linear equation [10].

Generally, if we simply use critical points as the start point for neural pathway directions, due to the nature of DT-MRI, a large number of critical points will be identified, and should we try to follow the streamlines from each critical point, an extremely confusing image would be produced. Figure 1 shows $256 \times 256 \times 60$ data from a healthy patient, with the critical points highlighted in red. Figure 2 shows an image of the results of calculating all the streamlines neighbouring the critical points found in Figure 1. Only vortex centers were selected for these critical points, and they do not include saddle points.

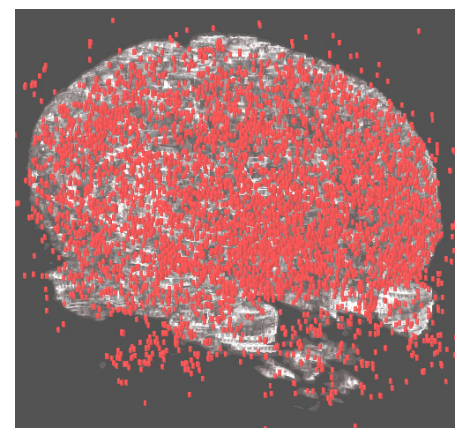

Figure 1: Searched critical points.

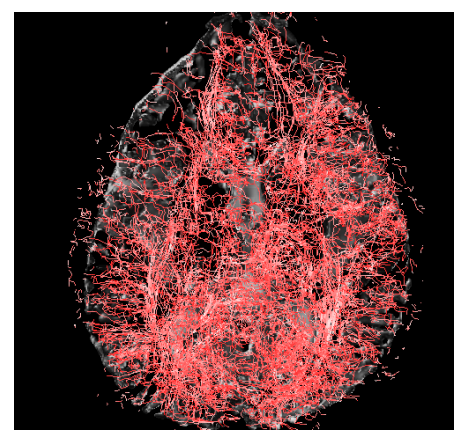

Figure 2: $\quad$ Streamlines from CPs. 


\section{Proposed method}

Our algorithm to follow neural pathways is shown in Figure 3. We assume that brain neural pathways are flow fields and calculate the vortex centers, and specify the locations with high FA values in that area as starting points of the streamlines. By displaying those streamlines that pass through the target region selected by the user, important neural pathways can be displayed in an easily understandable manner.

\subsection{Setting start points}

Critical points are important guides in understanding the characteristics of flow fields [8-10]. We take the centers of the 26 proximity voxels neighboring voxels containing critical points as candidates for the start points of streamlines. Out of these candidates, locations with high FA values (where there is a high possibility of a pathway being present) are set as start points of streamlines. Here, the FA value should be 0.5 or higher.

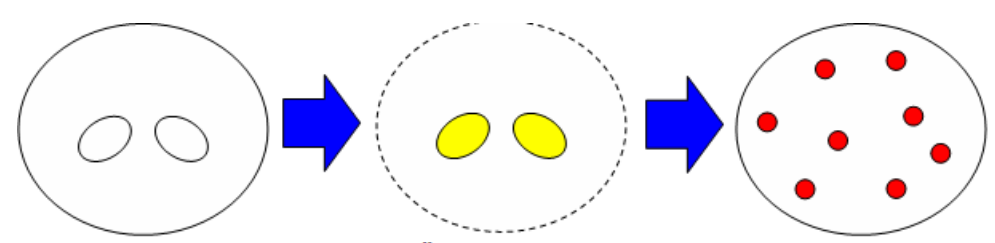

DT-MRI Segmentation Generation of Critical Points

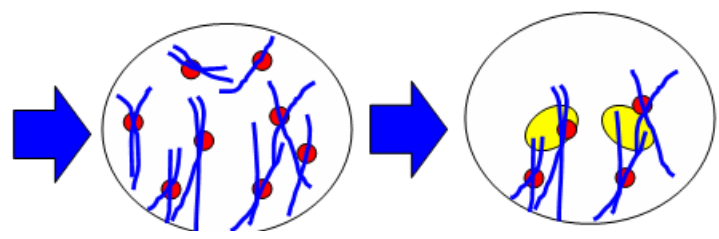

Generation of streamlines Selection of Neural Pathways

Figure 3: Overview of our algorithm.

\subsection{Setting target regions}

Following all the start points acquired in Step 3.1 results in an extremely high number of overlay pathways being extracted. The next step is thus to extract those pathways that pass through a specified region. To extract the necessary pathways, Sherbondy et al. set rectangular target regions, and only displayed those pathways that passed through these regions [6]. In addition to specifying rectangular regions, we extracted specified areas in the form of arbitrary multiple volume regions and designated them as target regions. These regions were extracted through a combination of a segmentation technique method such as the region-growing method and the interactive extraction method. 
Outlines of the crus posterius capsulae internae, cerebral peduncle and meningioma were extracted interactively by selecting a 2-dimensional $\mathrm{X}-\mathrm{Y}$ slice. The tool used was Volume Extractor Ver. 2.0 software that we have developed in our university [13]. Figure 4 shows that the neural pathway is selected by using a target region from neural pathways in Figure 2.
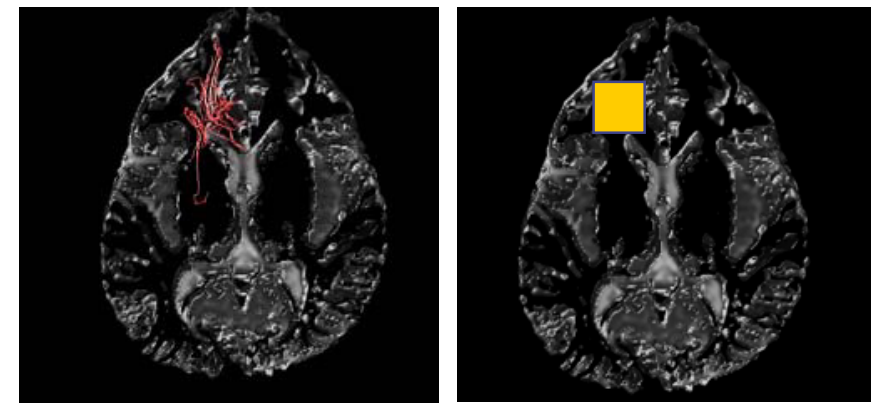

Figure 4: Selection of neural pathways using a target region (yellow box).

\subsection{Dealing with Intersections}

Of the 3 eigenvectors obtained, the vector that represents the primary direction alone is used for following the pathways. For this reason, when pathways intersect, only the pathway in the primary direction is followed, and intersecting pathways are sometimes not extracted.

In the method presented here, when an intersecting pathway is encountered, the direction just prior to the intersection is used, thus resolving this issue. In this manner, the angle of approach is undamaged and the intersecting pathway can be followed.

Note that intersecting points are determined by their low FA values $(0.2$ or less). Intersecting points feature multiple pathways in various directions, so the difference between eigenvalues decreases and FA value thus becomes smaller.

In addition, neural pathways are assumed to not suddenly turn sharp angles, and if the difference in angle between directions of the vector obtained directly before the intersection and the obtained direction is over a certain range $\left(60^{\circ}\right)$, the same process is applied.

\subsection{Coloration}

Generally, pathways are often color-coded according to direction or FA values. In such cases, differentiating pathways is difficult. There is a model of brain neural pathways that is used in hospitals to explain surgery to patients. Each region of neural pathways has been colored, making pathways easy to distinguish. To create this kind of brain neural pathway model, each target region is given a separate, distinguishable ID. Next, assuming that the pathways pass through multiple target regions, $2^{\mathrm{x}}-1$ tables are created containing the color or other identifying information (where $\mathrm{x}$ represents the number of selected target 
regions). For example, 2 target regions $\left(\mathrm{o}_{1}, \mathrm{o}_{2}\right)$ are used, and the pathways can be separated into 3 groups based on which target region they pass through, $\left(\mathrm{o}_{1}\right)$, $\left(\mathrm{o}_{2}\right)$ and $\left(\mathrm{o}_{1}, \mathrm{o}_{2}\right)$, and are assigned a number accordingly and colored. If 3 target regions $\left(\mathrm{o}_{1}, \mathrm{o}_{2}, \mathrm{o}_{3}\right)$ are used, then the combinations are $\left(\mathrm{o}_{1}\right),\left(\mathrm{o}_{2}\right),\left(\mathrm{o}_{3}\right),\left(\mathrm{o}_{1}, \mathrm{o}_{2}\right),\left(\mathrm{o}_{1}\right.$, $\left.\mathrm{o}_{3}\right),\left(\mathrm{o}_{2}, \mathrm{o}_{3}\right)$ and $\left(\mathrm{o}_{1}, \mathrm{o}_{2}, \mathrm{o}_{3}\right)$. A color is determined by referring to the table while assigning color information to each pathway number.

\section{Application and evaluation}

\subsection{Overview}

Two DT-MR images were used in this experiment, created by applying a magnetic field from 7 directions and the resolution is $256 \times 256 \times 60$. One DTMR image is of a healthy 19-year-old woman, and the other of a 53-year-old female patient with meningioma. The equipment used was an MR Scanner (GEMedical) with 3-T magnetic fields, and a gradient magnetic field factor $\mathrm{b}$ of 800 $\mathrm{s} / \mathrm{mm}^{2}$. Tri-linear interpolation was used to transform this data into $60 \times 60 \times 60$ isotropic voxels. Layered display of the cerebral nerve was performed using AVS/Express version 7.0 visualization software.

In Figure 2, streamlines from all critical points were pursued, making the important pathways impossible to distinguish. To resolve this issue, target regions through which important brain neural pathways pass are set. In this paper, the crus posterius capsulae internae and meningioma areas were selected as target regions. Of the pathway groups pursued from near critical points, only those passing through the target region (crus posterius capsulae internae) were displayed (Figure 5). Pathways in Figure 5 are not color-coded by FA value, but by whether they pass through the left or right crus posterius capsulae internae.

Figure 6 shows an MRI slice image from a meningioma patient, with the white area on the left identified as an abnormality. In Figure 7, the region around the meningioma was selected as the target region, and only those pathways passing through this region were displayed. These are superimposed on MRI, and the area around the tumor is color-coded yellow, making it easier to grasp the relative locations of the brain and tumor.

\subsection{Computational processing time}

This section describes the processing time taken by this method. The operating system used in this research was Windows XP, running Visual $\mathrm{C}++$ version 6.0. The hardware used consisted of a $1.50 \mathrm{GHz}$ Pentium M CPU with $1.25 \mathrm{~GB}$ of RAM. Eigenvalues and eigenvectors were calculated using the Jacobi method [12]. Three types of processing time comparison were performed: processing time by specified regions with neural pathways passing through; processing time by data size; and processing time when critical points are used as starting points and when they are not. The left and right of the crus posterius capsulae internae and cerebral peduncle of the healthy patient were selected as target regions. With data obtained from the meningioma patient, the area around the tumor was selected. 

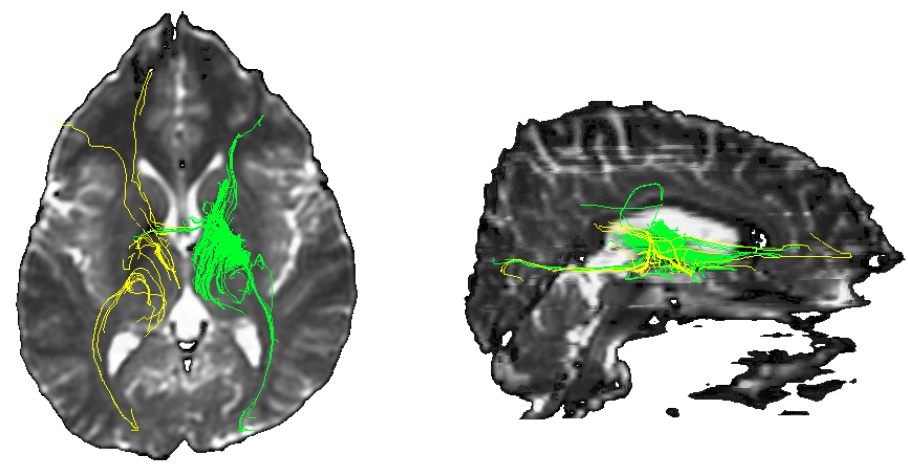

Figure 5: Coloration for brain pathway using target regions ( $\mathrm{xy}$ and $\mathrm{yz}$ plane).
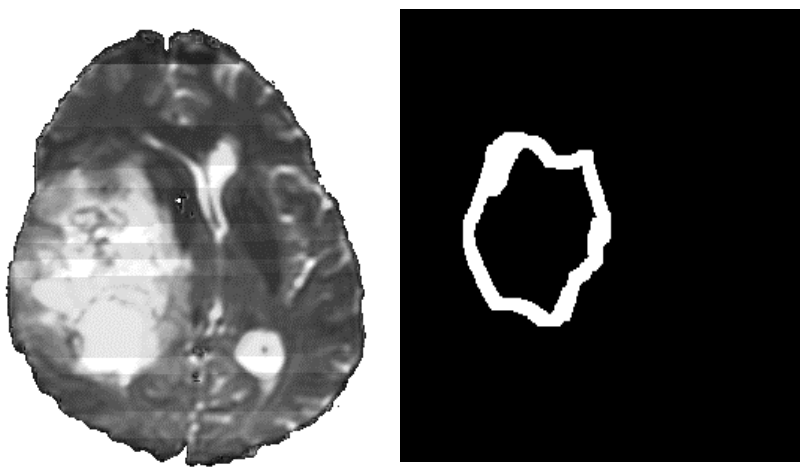

Figure 6: $\quad$ MRI of meningioma patient and a target region.
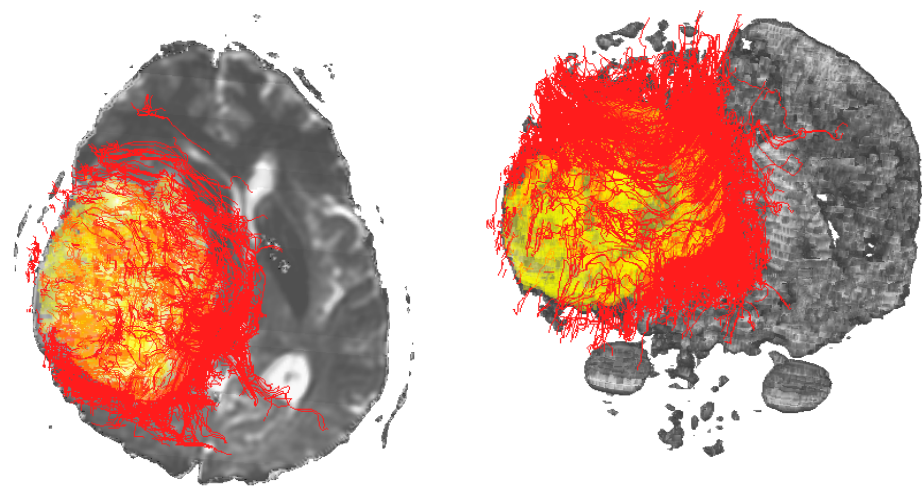

Figure 7: $\quad$ Pathway pursue results (xy plane and 3D display). 
Table 1: A comparison of computational time.

\begin{tabular}{|l|l|l|c|c|c|}
\hline & Starting Point & Target Region & $\begin{array}{c}\text { Pursuit } \\
\text { Time }\end{array}$ & Other & Total \\
\hline a & Crus posterius & None & 4.797 & 13.457 & 18.254 \\
\hline a & Critical point & Crus posterius & 60.984 & 49.631 & 110.615 \\
\hline a & $\begin{array}{l}\text { Cerebral } \\
\text { peduncle }\end{array}$ & None & 4.133 & 13.301 & 17.434 \\
\hline a & Critical point & Cere peduncle & 44.754 & 49.875 & 94.629 \\
\hline b & Crus posterius & None & 0.150 & 1,034 & 1.184 \\
\hline b & Critical point & Crus posterius & 1.579 & 5.832 & 7.410 \\
\hline b & Cere. peduncle & None & 0.170 & 0.874 & 1.044 \\
\hline b & Critical point & Cere. peduncle & 0.921 & 4.830 & 5.751 \\
\hline c & Crus posterrius & None & 102.381 & 56.019 & 158.400 \\
\hline c & Critical point & Crus posterrius & 505.053 & 164.895 & 669.948 \\
\hline c & Cere. peduncle & None & 97.467 & 56.384 & 153.851 \\
\hline c & Critical point & Cere. peduncle & 410.393 & 165.759 & 576.152 \\
\hline d & Tumor area & None & 2301.456 & 68.585 & 2370.041 \\
\hline d & Critical point & Tumor area & 1136.915 & 55.432 & 1192.347 \\
\hline e & Tumor area & None & 3.945 & 2.948 & 6.893 \\
\hline e & Critical point & Tumor area & 7.147 & 5.538 & 12.685 \\
\hline & Tumor area & None & 22322.270 & 238.684 & 22560.957 \\
\hline & Critical point & Tumor area & 14285.287 & 170.990 & 14456.277 \\
\hline & f & & & \\
\hline
\end{tabular}

Table 1 shows computational time for each set of data in which the target region is set. The "Crus posterius", "Cere. Peduncle", and "Tumor area" represent Crus posterius capsulae internae, Cerebral peduncle, and Area around tumor (meningioma), respectively. As a pre-process, the diffusion direction of each pixel is deduced from the acquired DT-MRI and written to a file. After reading the file, all critical points are calculated. The extracted neural pathways are written in an external file. The time taken to pursue neural pathways and all other times (reading and writing files, extracting critical points) were totalized separately. All times are measured in seconds. The alphabetic code assigned in Table 1 corresponds to the following resolutions and patient data. The third number shows CPU time searching critical points (second). The forth number shows the number of critical points. 
- a: 256x256x60 data from healthy patient: $32.1 \mathrm{sec} ., 6448$

- b: 60x60x60 data from healthy patient: $3.8 \mathrm{sec} ., 405$

- c: $256 \times 256 \times 240$ data of healthy patient: 101.0 sec., 20037

- d: $256 \times 256 \times 60$ data of meningioma patient: 27.2 sec., 5148

- e: $60 \times 60 \times 60$ data of meningioma patient: $3.5 \mathrm{sec}$., 401

- f: $256 \times 256 \times 240$ data of meningioma patient: $92.8 \mathrm{sec}$., 17757

As shown in Table 1, if the selected target region is small, pursuing neural pathways directly from the target regions is more effective, rather than pursuit from the critical points. However, when the target region is large, as with the area around the meningioma, pursuing the neural pathways becomes increasingly time-consuming, and extracting those neural pathways from around the critical points is faster. We can see that a large number of critical points are created, if image data includes noise. By only selecting the important critical points ahead of time, calculation time can be reduced. Target regions were interactively specified as 3-dimensional target areas (volume region). Segmentation of the crus posterius capsulae internae and cerebal peduncle, as in this example, can be relatively clearly imaged using MRI, and since the number of slices is low, its extraction is relatively easy.

\section{Conclusion}

In this research, pathways were pursued using critical points as starting points, and pathways passing through target regions were extracted. Using the proposed coloration method, neural pathways are easily distinguished, such as by colorcoding the left and right crus posterius capsulae internae separately (Figure 5). In addition, data from a patient with a meningioma were used in this paper to show the potential usefulness of this method in surgical planning by setting an area around the tumor as a target region and pursuing the identified neural pathways. Areas include parts that appeared to be meningioma. In Figure 7, pathways avoiding the meningioma were extracted, and we can presume that accurate pursuit results were obtained. If the area of the target region is large, then processing capacity is improved using this method compared to pursuing neural pathways from the target region. However, if the target region is small, such as with the crus posterius capsulae internae, then extracting pathways directly from the target region may be faster. In such cases, pathway pursuit results do not differ greatly from the method using critical points.

Considering the future prospects of this method, pathway pursuit results do not seem accurate enough, and more accurate extraction of neural pathways from points where pathways intersect is needed. In addition, to reduce the burden placed on the user, the current methods of manual segmentation must be automated. In this case, this method was applied to the area around the meningioma, and the region around the borderline could be clarified. In future, this method might be applied to predicting border areas of gliomas where such detection is difficult, based on the direction of neural pathways. In addition, interrelationships between neural pathways and blood vessels and the visualization of this relationship remains a very interesting issue. 


\section{Acknowledgements}

We would like to extend our deep thanks to Mr. Yoshiyuki Kanbara of Iwate Medical University Isotope Center for the many words of advice and for cooperation in gathering data. We would also likely to sincerely thank Prof. Koji Koyamada and Prof. Koji Sakai of Kyoto University for their constant and valuable opinions and advice.

\section{References}

[1] Aoki, S., Abe, O., \& Masutani, Y., Korede wakaru Difusion MRI, Shueisha, 2002.

[2] Haines, D. E. (Yamauchi, A. Translation), Haines Neural Pathway Anatomy Atlas Second-edition, Medical Science International, 2003.

[3] Barr, H. A., Oriented Tensor Reconstruction: Tracing Neural Pathways from Diffusion Tensor MRI, IEEE Visualization 2002, pp.387-394, 2002.

[4] Basser, P. J., Pajevic, S., Pierpaoli, C., \& Aldroubi, A., Fiber tract following in the human brain using DT-MRI data, IEICE Trans Inf Sys, E85D, pp. 15-21, 2002.

[5] Volume-One, http://www.volume-one.org/dTV

[6] Sherbondy, A., Akers, D., Macenzie, R., Doughety, R., \& Wandell, B., Exploring Connectivity of the Brain's White Matter with Dynamic Queries, IEEE Trans. on Vis. and Computer Graphics, Vol. 11, No. 4, pp. 419-430, 2005.

[7] Masutani, Y., Aoki, S., Abe, O., \& Otomo, K., White matter fiber tracking based on interpolation vector of main diffusion tensor with high anisotropic property, Annual Meeting 2004 of Japanese Society of Medical Imaging Technology (JAMIT), 2004 (in Japanese).

[8] Sakai, K., A study of visualization techniques based on critical points, Iwate Prefectural University, Faculty of information and computational science, doctorial paper, 2004.

[9] Fujimura, H., Sai Y., Mochizuki T., Doi, A., Inoue, T., Sasaki, M. \& Ogawa, A., Visualization o f brain neural pathway by using streamlines, Japan Visualization Symposium, Vol. 25, No. 1, pp.1-8, 2005.

[10] Doi, A., Suzuki, S., Koyamada K. \& Sannakanishi, S., Vortex flow visualization using tetrahedral subdivision - Improved method and its applications, Journal of gazo denshi gakkai, Vol. 26, No. 4, pp. 333-340, 1997.

[11] Hinohara, S., Abe, M., Asami, K. \& Seki, Y., Systematic nursing art and science lecture special basic 1, Human structure and its functions, anatomy and physiology, Igaku-shoin, 1999.

[12] Press, W. H., Flannency, B. P., Teukolsky, S. A. \& Vetterling, W. T., Numerical Recipes in C: The Art of Scientific Computing, Cambridge University Press, 2004.

[13] Sirius, http://www.dicult.com/sirius/ 